\title{
Consumo alimentar conforme o tipo de alimentação consumida em escolas de zona rural no Sul do Brasil
}

\author{
Food intake according to the type of food consumed in schools \\ in a rural area in southern Brazil
}

Caren Taiane Radtke Bubolz ${ }^{1}$

Airton José Rombaldi ${ }^{2}$

Nicole Gomes Gonzales ${ }^{2}$

Mario Renato Azevedo ${ }^{2}$

Samanta Winck Madruga ${ }^{1}$

${ }^{1}$ Departamento de Nutrição, Faculdade de Nutrição, Universidade Federal de Pelotas (UFPel), R. Gomes Carneiro 1, Centro. 96010-610 Pelotas RS Brasil. carenbubolz@hotmail.com ${ }^{2}$ Departamento de Desportos, Escola Superior de Educação Física, UFPel. Pelotas RS Brasil.
Abstract The scope of the article was to evaluate food consumption according to the type of food consumed in rural schools. A cross-sectional descriptive study was conducted among adolescents enrolled in 12 rural public schools in Canguçu in the state of Rio Grande do Sul, Brazil. The research was linked to the intervention named "Physical Education +: Practicing Health in School." Dietary intake was evaluated using consumption markers from the Brazilian Food and Nutrition Surveillance System. The main independent variable was the type of food consumed at school (brought from home, provided by the school free of charge and/or purchased at school). Descriptive statistics and the Chi-square test were used and the significance level was $p<0.05$. The study enlisted 526 students, $51.3 \%$ female and $88.0 \%$ consuming food free of charge. From 15\% to $31 \%$ of adolescents consumed foods considered unhealthy for more than three days in the preceding week. Students who reported taking a snack to school consumed raw salad, fresh fruit or fruit salad, biscuits/crackers and packet snacks and soft drinks more frequently. It was concluded that the consumption of healthy foods among schoolchildren in rural areas was low and interventions are needed to improve the current situation of inadequate feeding practices.

Key words Adolescent, Food consumption, School food, Rural population
Resumo Objetivou-se avaliar o consumo alimentar de escolares conforme o tipo de alimentação consumida em escolas de zona rural. Trata-se de um estudo transversal realizado com adolescentes de 12 escolas públicas rurais de Canguçu/ RS. A investigação está vinculada à intervenção "Educação Física +: Praticando Saúde na Escola". O consumo alimentar foi avaliado pelos marcadores do Sistema de Vigilância Alimentar e Nutricional. A variável independente principal foi o tipo de alimentação consumida (trazida de casa, fornecida gratuitamente e/ou comprada na escola). Foi utilizada a estatística descritiva e o teste de Qui-quadrado com nível de significância de $p<0,05$. Participaram do estudo 526 escolares, $51,3 \%$ do sexo feminino e $88,0 \%$ que consumiram a alimentação gratuita. Entre 15-31\% dos adolescentes consumiram mais de três dias na semana anterior alimentos considerados não saudáveis. Os escolares que relataram levar lanche de casa para a escola consumiram com maior frequência salada crua, frutas frescas ou salada de frutas, bolachas/biscoitos salgados e salgadinhos de pacote e refrigerante. Concluiu-se que o consumo de alimentos saudáveis entre os escolares da zona rural foi baixo e intervenções são necessárias a fim de melhorar o quadro atual de práticas inadequadas de alimentação.

Palavras-chave Adolescente, Consumo de alimentos, Alimentação escolar, População rural 


\section{Introdução}

A adolescência é um período que compreende a faixa etária dos 10 aos 19 anos de idade ${ }^{1}$. É nesse momento que acontecem intensas e profundas transformações nas quais o indivíduo está definindo e aprimorando sua identidade, estilo de vida e desenvolvendo preocupações ligadas ao corpo e à aparência. Essas características atuam como determinantes do hábito e do comportamento alimentar do adolescente $e^{2-4}$.

Contudo, as alterações no estilo de vida, em particular do padrão alimentar, estão provocando efeitos sobre a composição corporal e a saú$\mathrm{de}^{5,6}$, principalmente nos adolescentes, visto que as descrições das práticas alimentares adotadas nesta fase da vida caracterizam-se pela baixa ingestão de frutas e hortaliças e preferência por dietas ricas em gorduras, açúcares e sódio, cujo excesso relaciona-se com o aumento da ocorrência de obesidade e de suas principais comorbidades $^{6-9}$.

Considerando o exposto, a fase da adolescência pode ser definidora dos hábitos alimentares ao longo do ciclo vital e os estudos vêm mostrando sistematicamente que esse consumo está inadequado ${ }^{10-12}$. Nesse sentido, considera-se o ambiente escolar como importante meio para a educação alimentar e nutricional ${ }^{13,14}$. Entretanto, é conhecido que os alimentos comercializados pelas cantinas escolares contêm elevada densidade energética, cujo consumo frequente pode contribuir para a prevalência de excesso de peso ${ }^{15,16}$.

A presença das cantinas nas escolas ainda parece interferir na adesão ao Programa Nacional de Alimentação Escolar (PNAE) ${ }^{15,16}$, o qual visa atender às necessidades nutricionais dos estudantes durante sua permanência em sala de aula, contribuindo para o crescimento, o desenvolvimento e a aprendizagem dos mesmos, bem como para a formação de bons hábitos alimentares ${ }^{17}$, podendo representar uma forte limitação à educação alimentar e nutricional ${ }^{15}$.

Neste sentido, este estudo teve como objetivo avaliar o consumo alimentar de adolescentes escolares e sua relação com o tipo de alimentação escolar consumida.

\section{Métodos}

Trata-se de um estudo transversal, desenvolvido com escolares de ambos os sexos, de $5^{\mathrm{a}}$ a $8^{\mathrm{a}}$ séries, da rede pública de ensino da zona rural no município de Canguçu, localizado ao sul do estado do Rio Grande do Sul, o qual caracteriza-se por atividades predominantemente agrícolas. $\mathrm{O}$ município possui aproximadamente 56 mil habitantes, com $63 \%$ residindo na zona rural ${ }^{11}$.

Esta investigação está vinculada ao projeto de pesquisa "Educação Física +: Praticando Saúde na Escola $(\mathrm{EF}+)$ ". Trata-se de uma intervenção elaborada pelo Grupo de Estudos em Epidemiologia da Atividade Física do Programa de Pós-graduação em Educação Física da Universidade Federal de Pelotas, desenvolvida no ambiente escolar que objetivou aumentar a prática de atividade física e o conhecimento da relação entre estilo de vida saudável (incluindo a prática de atividade física e a alimentação saudável) e o grau de saúde dos jovens. Apostilas foram desenvolvidas para cada ano escolar, do $6^{\circ}$ ano do ensino fundamental ao $3^{\circ}$ ano do ensino médio, estruturadas em capítulos e organizadas da seguinte forma: texto de apoio, planos de aula, informações complementares e sugestão de avaliação ${ }^{18}$.

Por questões logísticas e financeiras, não foi possível realizar a coleta de dados com todas as escolas da zona rural do município de Canguçu/ RS. Nesse sentido, para o presente estudo foram selecionadas 12 escolas rurais, de um total de 39, definidas por critério de amostragem estratificada de acordo com o tamanho e a rede de ensino (municipal/estadual). No início do ano letivo de 2012, os alunos das escolas selecionadas foram medidos em relação ao nível de atividade física, frequência de consumo alimentar e nível de conhecimento sobre atividade física e saúde, sendo que tais dados compuseram a linha de base do estudo e foram utilizados neste artigo.

O consumo alimentar dos escolares foi avaliado de forma autoaplicada através do Formulário de Marcadores do Consumo Alimentar do Sistema de Vigilância Alimentar e Nutricional (SISVAN) para indivíduos com cinco anos de idade ou mais, proposto pelo Ministério da Saúde ${ }^{19}$. Este instrumento objetiva identificar a frequência de consumo de alguns alimentos e/ ou bebidas que estão relacionados tanto a uma alimentação saudável como não saudável, no período de sete dias anteriores à entrevista ${ }^{17}$.

Os alimentos que compuseram o instrumento foram: salada crua, legumes e verduras cozidos, frutas frescas ou salada de frutas, feijão, leite ou iogurte, batata frita, batata de pacote e salgados fritos, hambúrguer e embutidos, bolachas/ biscoitos salgados ou salgadinhos de pacote, bolachas/biscoitos doces ou recheados, doces, balas e chocolates e refrigerante. A análise do consumo 
de cada um dos alimentos foi operacionalizada em dois grupos: até três dias e quatro dias ou mais de consumo nos sete dias anteriores à entrevista. A variável independente principal foi o tipo de alimentação consumida na escola (trazida de casa, fornecida pela escola gratuitamente e/ ou comprada na escola).

Atuaram como entrevistadores, três alunos do curso de mestrado em Educação Física da ESEF/ UFPel (dois homens e uma mulher) e outros três alunos (dois homens e uma mulher) do curso de graduação em Educação Física - Licenciatura, da mesma instituição, os quais receberam um treinamento de oito horas sobre o instrumento, como aplicá-lo e a conduta a ser observada no momento da entrevista.

Os dados foram duplamente digitados no programa Epi Info 6.0. As análises estatísticas foram realizadas no programa STATA versão 12.0, sendo utilizado o teste de Qui-quadrado para verificar a associação entre tipo de alimentação consumida pelos escolares e variáveis nutricionais. Os dados foram apresentados com IC95\% e o nível de significância aceito foi de $\mathrm{p}<0,05$. O estudo foi aprovado pelo Comitê de Ética em Pesquisa com seres humanos da Escola Superior de Educação Física, da Universidade Federal de Pelotas, credenciado junto ao Conselho Nacional de Saúde. Os adolescentes (maiores de 18 anos) e os pais/responsáveis (para os menores de 18 anos) assinaram o Termo de Consentimento Livre e Esclarecido anterior à sua inclusão na coleta de dados e em todas as fases da pesquisa, foram garantidos o anonimato, a liberdade de recusa e a possibilidade de retirada do consentimento.

\section{Resultados}

Participaram do estudo 526 escolares da zona rural da cidade de Canguçu/RS, no ano de 2012, com idade média de 12,9 anos (DP $\pm 1,5$ anos), sendo a maioria do sexo feminino $(51,3 \%)$, cursando a $5^{\mathrm{a}-6^{\mathrm{a}}}$ séries $(59,1 \%)$, pertencente à rede municipal de ensino $(83,6 \%)$ e consumindo a alimentação oferecida pela própria escola $(88,0 \%)$ (Tabela 1). Além disto, a maioria dos alunos que não comia a refeição fornecida pelas escolas, comprava lanches na cantina/bar existente nas instituições (76,3\%).

A análise dos marcadores de consumo alimentar dos escolares nos últimos sete dias está demonstrada na Tabela 2. O marcador de alimentação saudável mais frequente (quatro dias
Tabela 1. Descrição dos escolares de ensino fundamental de escolas públicas rurais do município de Canguçu/RS - Brasil, 2012.

\begin{tabular}{|c|c|c|}
\hline \multirow{2}{*}{ Variáveis } & \multicolumn{2}{|c|}{ Total } \\
\hline & $\mathbf{N}$ & $\%$ \\
\hline \multicolumn{3}{|l|}{ Idade $($ anos $)(\mathrm{n}=520)$} \\
\hline $10-12$ & 225 & 43,3 \\
\hline $13-15$ & 267 & 51,3 \\
\hline 16 ou mais & 28 & 5,4 \\
\hline \multicolumn{3}{|l|}{ Sexo $(n=526)$} \\
\hline Masculino & 256 & 48,7 \\
\hline Feminino & 270 & 51,3 \\
\hline \multicolumn{3}{|l|}{ Série $(n=526)$} \\
\hline $5^{a}$ & 164 & 31,2 \\
\hline $6^{\mathrm{a}}$ & 147 & 27,9 \\
\hline $7^{a}$ & 95 & 18,1 \\
\hline $8^{a}$ & 120 & 22,8 \\
\hline \multicolumn{3}{|l|}{ Rede de ensino $(n=526)$} \\
\hline Municipal & 440 & 83,6 \\
\hline Estadual & 86 & 16,4 \\
\hline \multicolumn{3}{|l|}{ Tipo de merenda } \\
\hline \multicolumn{3}{|l|}{ Casa $(\mathrm{n}=514)$} \\
\hline Sim & 93 & 18,1 \\
\hline Não & 421 & 81,9 \\
\hline Escola $(n=499)$ & 439 & 88,0 \\
\hline Sim & 60 & 12,0 \\
\hline \multicolumn{3}{|l|}{ Não } \\
\hline Cantina ou bar $(\mathrm{n}=510)$ & 389 & 76,3 \\
\hline Sim & 121 & 23,7 \\
\hline Não & & \\
\hline
\end{tabular}

ou mais) foi o feijão (79,8\%), seguido do leite ou iogurte $(40,8 \%)$. Apenas $31,2 \%$ dos escolares consumiram frutas frescas ou salada de frutas na maioria dos dias da semana e quase a totalidade dos estudantes avaliados consumiram legumes e verduras cozidos e salada crua menos de quatro dias na última semana $(89,4 \%)$.

Foi observado que uma expressiva parcela dos adolescentes consumiu por mais de quatro dias na semana anterior alimentos considerados não saudáveis como hambúrguer e embutidos $(15,2 \%)$, batata frita ou de pacote e salgados fritos $(17,1 \%)$, refrigerante $(18,6 \%)$, bolachas/ biscoitos salgados $(27,4 \%)$ e bolachas/biscoitos doces (31\%).

Na Tabela 3, estão apresentados os dados referentes aos marcadores de consumo alimentar conforme o tipo de alimentação consumida na escola. Verificou-se que os escolares que levavam lanche de casa para a escola consumiram, na maioria dos dias da semana anterior, alimen- 
Tabela 2. Consumo alimentar nos últimos sete dias, de escolares de ensino fundamental de escolas públicas do município de Canguçu/RS - Brasil, 2012 ( $\mathrm{n}=526)$.

\begin{tabular}{|c|c|c|}
\hline \multirow{2}{*}{ Variáveis } & \multicolumn{2}{|r|}{ Total } \\
\hline & $\mathbf{N}$ & Percentual (\%) \\
\hline \multicolumn{3}{|l|}{ Salada crua } \\
\hline Até 3 dias & 448 & 88,7 \\
\hline 4 ou mais dias & 57 & 11,3 \\
\hline \multicolumn{3}{|l|}{ Legumes e verduras cozidos } \\
\hline Até 3 dias & 447 & 89,4 \\
\hline 4 ou mais dias & 53 & 10,6 \\
\hline \multicolumn{3}{|l|}{ Frutas frescas ou salada de frutas } \\
\hline Até 3 dias & 346 & 68,8 \\
\hline 4 ou mais dias & 157 & 31,2 \\
\hline \multicolumn{3}{|l|}{ Feijão } \\
\hline Até 3 dias & 102 & 20,2 \\
\hline 4 ou mais dias & 404 & 79,8 \\
\hline \multicolumn{3}{|l|}{ Leite ou iogurte } \\
\hline Até 3 dias & 295 & 59,2 \\
\hline 4 ou mais dias & 203 & 40,8 \\
\hline \multicolumn{3}{|l|}{ Batata frita, batata de pacote e salgados fritos } \\
\hline Até 3 dias & 416 & 82,9 \\
\hline 4 ou mais dias & 86 & 17,1 \\
\hline \multicolumn{3}{|l|}{ Hambúrguer e embutidos } \\
\hline Até 3 dias & 425 & 84,8 \\
\hline 4 ou mais dias & 76 & 15,2 \\
\hline \multicolumn{3}{|c|}{ Bolachas/biscoitos salgados ou salgadinhos de pacote } \\
\hline Até 3 dias & 363 & 72,6 \\
\hline 4 ou mais dias & 137 & 27,4 \\
\hline \multicolumn{3}{|c|}{ Bolachas/biscoitos doces ou recheados ou doces, balas, chocolates } \\
\hline Até 3 dias & 340 & 69,0 \\
\hline 4 ou mais dias & 153 & 31,0 \\
\hline \multicolumn{3}{|l|}{ Refrigerante } \\
\hline Até 3 dias & 399 & 81,4 \\
\hline 4 ou mais dias & 91 & 18,6 \\
\hline
\end{tabular}

tos considerados saudáveis como salada crua $(17,8 \% ; \mathrm{p}<0,03)$ e frutas frescas ou salada de frutas $(42,4 \% ; \mathrm{p}<0,01)$. Apesar das associações saudáveis indicadas acima, houve também associações não saudáveis, pois quem trouxe lanche de casa consumiu mais alimentos como bolachas/biscoitos salgados ou salgadinhos de pacote $(36,3 \% ; \mathrm{p}<0,04)$ e refrigerante $(26,4 \%$; $\mathrm{p}<$ $0,04)$.

\section{Discussão}

O presente estudo apresenta uma avaliação de escolares unicamente da zona rural, fato que merece destaque uma vez que são escassos os estu- dos de consumo alimentar com esta população. Pontos positivos que podem ser ressaltados na pesquisa são a seleção das escolas e número de respondentes, além da padronização dos métodos de coleta de dados, treinamento adequado dos entrevistadores e o instrumento utilizado.

Os estudos brasileiros que investigaram o consumo alimentar de adolescentes têm utilizado uma grande variedade de metodologias para avaliação, optando, frequentemente, pela aplicação de Recordatórios de 24 horas ou Questionários de Frequência Alimentar (QFA), o que indica cautela na comparação dos resultados entre os estudos, visto que instrumentos autoaplicados (como muitos QFAs), os quais coletam um menor número de informações, podem não repre- 
Tabela 3. Consumo alimentar conforme o tipo de merenda consumida por escolares do município de Canguçu/RS Brasil, $2012(\mathrm{n}=526)$.

\begin{tabular}{|c|c|c|c|c|c|c|}
\hline $\begin{array}{l}\text { Alimentos do formulário de } \\
\text { marcadores do consumo alimentar }\end{array}$ & $\begin{array}{l}\text { Traz lanche de } \\
\text { casa }(\mathbf{n}=93) \\
\%\left(\mathrm{IC}_{95 \%}\right)\end{array}$ & $\begin{array}{l}\text { Valor } \\
\mathrm{p}^{*}\end{array}$ & $\begin{array}{c}\text { Consome } \\
\text { merenda da } \\
\text { escola }(n=439) \\
\%\left(\mathrm{IC}_{95 \%}\right)\end{array}$ & $\begin{array}{c}\text { Valor } \\
\mathbf{p}^{*}\end{array}$ & $\begin{array}{c}\text { Compra na } \\
\text { cantina ou bar da } \\
\text { escola }(\mathbf{n}=389) \\
\%\left(\mathrm{IC}_{95 \%}\right)\end{array}$ & $\begin{array}{c}\text { Valor } \\
p^{*}\end{array}$ \\
\hline $\begin{array}{l}\text { Salada crua }(\mathrm{n}=505) \\
\text { Até } 3 \text { dias } \\
4 \text { ou mais dias }\end{array}$ & $\begin{array}{c}82,2(71,8-90,3) \\
17,8(4,0-45,6)\end{array}$ & $<0,03$ & $\begin{array}{c}88,7(85,2-91,8) \\
11,3(4,6-24,8)\end{array}$ & 0,9 & $\begin{array}{c}88,0(84,2-91,4) \\
12,0(4,9-26,3)\end{array}$ & 0,4 \\
\hline $\begin{array}{l}\text { Legumes e verduras cozidos }(\mathrm{n}=500) \\
\text { Até } 3 \text { dias } \\
4 \text { ou mais dias }\end{array}$ & $\begin{array}{c}86,2(76,8-93,4) \\
13,8(2,1-48,4)\end{array}$ & 0,3 & $\begin{array}{c}89,5(86,1-92,5) \\
10,5(3,7-24,1)\end{array}$ & 0,9 & $\begin{array}{c}89,5(85,6-92,5) \\
10,5(2,8-23,7)\end{array}$ & 0,9 \\
\hline $\begin{array}{l}\text { Frutas frescas ou salada de frutas } \\
(\mathrm{n}=503) \\
\quad \text { Até } 3 \text { dias } \\
4 \text { ou mais dias }\end{array}$ & $\begin{array}{l}57,6(44,1-71,9) \\
42,4(27,8-60,4)\end{array}$ & $<0,01$ & $\begin{array}{l}69,2(63,7-74,4) \\
30,8(23,1-39,4)\end{array}$ & 0,7 & $\begin{array}{c}66,8(6,1-72,5) \\
33,2(25,0-42,0)\end{array}$ & 0,1 \\
\hline $\begin{array}{r}\text { Feijão }(\mathrm{n}=506) \\
\text { Até } 3 \text { dias } \\
4 \text { ou mais }\end{array}$ & $\begin{array}{l}21,7(5,7-43,7) \\
78,3(66,4-86,7)\end{array}$ & 0,6 & $\begin{array}{l}20,2(12,6-30,4) \\
79,8(75,2-83,9)\end{array}$ & 1,0 & $\begin{array}{l}20,4(12,0-30,8) \\
79,6(74,6-83,9)\end{array}$ & 0,8 \\
\hline $\begin{array}{l}\text { Leite ou iogurte }(\mathrm{n}=498) \\
\text { Até } 3 \text { dias } \\
4 \text { ou mais dias }\end{array}$ & $\begin{array}{l}52,7(37,2-66,7) \\
47,3(31,2-62,3)\end{array}$ & 0,2 & $\begin{array}{l}57,7(51,5-64,1) \\
42,3(35,2-50,1)\end{array}$ & 0,2 & $\begin{array}{l}58,6(51,8-65,1) \\
41,4(33,6-49,5)\end{array}$ & 0,6 \\
\hline $\begin{array}{l}\text { Hambúrguer e embutidos }(\mathrm{n}=501) \\
\text { Até } 3 \text { dias } \\
4 \text { ou mais dias }\end{array}$ & $\begin{array}{c}82,2(71,8-90,3) \\
17,8(4,0-45,6)\end{array}$ & 0,5 & $\begin{array}{c}86,0(82,0-89,3) \\
14,0(5,9-24,6)\end{array}$ & 0,2 & $\begin{array}{c}84,0(79,6-87,9) \\
16,0(8,2-28,1)\end{array}$ & 0,4 \\
\hline $\begin{array}{l}\text { Bolachas/biscoitos salgados ou } \\
\text { salgadinhos de pacote }(\mathrm{n}=500) \\
\text { Até } 3 \text { dias } \\
4 \text { ou mais dias }\end{array}$ & $\begin{array}{l}63,7(50,1-76,0) \\
36,3(20,4-54,9)\end{array}$ & $<0,04$ & $\begin{array}{l}74,0(68,7-78,6) \\
26,0(18,1-35,0)\end{array}$ & 0,1 & $\begin{array}{l}71,9(66,2-77,1) \\
28,1(19,8-37,5)\end{array}$ & 0,6 \\
\hline $\begin{array}{l}\text { Bolachas/biscoitos doces ou recheados } \\
\text { ou doces, balas, chocolates }(\mathrm{n}=493) \\
\text { Até } 3 \text { dias } \\
4 \text { ou mais dias }\end{array}$ & $\begin{array}{l}61,5(46,7-73,5) \\
38,5(21,5-55,1)\end{array}$ & 0,09 & $\begin{array}{l}68,3(62,7-73,7) \\
31,7(24,1-40,4)\end{array}$ & 0,6 & $\begin{array}{l}66,7(60,6-72,6) \\
33,3(25,4-42,6)\end{array}$ & 0,06 \\
\hline $\begin{array}{l}\text { Refrigerante }(\mathrm{n}=490) \\
\text { Até } 3 \text { dias } \\
4 \text { ou mais dias }\end{array}$ & $\begin{array}{l}73,6(60,9-83,7) \\
26,4(10,2-48,4)\end{array}$ & $<0,04$ & $\begin{array}{l}81,6(76,9-85,4) \\
18,4(10,2-28,3)\end{array}$ & 0,6 & $\begin{array}{r}79,6(74,4-83,9) \\
20,4(11,5-30,5) \\
\end{array}$ & 0,07 \\
\hline
\end{tabular}

* Teste de Qui-quadrado de Pearson

sentar de forma real o comportamento alimentar do indivíduo avaliado.

O presente estudo utilizou o Formulário de Marcadores do Consumo Alimentar para indivíduos com cinco anos de idade ou mais do SISVAN. Este instrumento, embora não meça hábito alimentar, é proposto pelo Ministério da Saúde ${ }^{19}$ e tem como objetivo monitorar o padrão alimentar e o estado nutricional da população, sendo adequado para identificar indivíduos ou grupos que apresentem agravos e riscos para saúde, contribuindo para o conhecimento da natureza e da magnitude dos problemas de nutrição $0^{20}$.
Neste estudo, a maioria dos estudantes consumiu a alimentação fornecida pela escola $(88,0 \%)$ ou comprou lanche na cantina escolar (76,3\%). Parte dos alimentos comercializados nas cantinas escolares como balas, pirulitos, biscoitos doces ou salgados, salgados fritos e refrigerantes contém alta densidade calórica, elevado teor de açúcar, sódio e gorduras, além de serem pobres em fibras e micronutrientes (vitaminas e minerais) e possuírem aditivos e corantes. Esses, são considerados alimentos não saudáveis e entende-se que quando a escola oferece esse tipo de alimento, está incentivando o consumo dos mesmos ${ }^{21}$. 
Os achados deste estudo mostram que os alimentos considerados saudáveis foram pouco consumidos por escolares adolescentes de escolas públicas rurais de Canguçu/RS e, com exceção do consumo de feijão $(79,8 \%)$, os demais alimentos saudáveis foram relatados por menos da metade dos adolescentes (entre 10,6 e 40,8\%). Estudos anteriores, um realizado na zona rural com escolares adolescentes e seus pais em Barão do Triunfo/RS ${ }^{22}$ e outro conduzido na zona urbana, realizado com adolescentes em Pelotas/ $\mathrm{RS}^{23}$, mostraram resultados semelhantes. Esse fato chama a atenção e refuta a hipótese inicial do presente estudo, uma vez que o esperado era que essa população, por ser essencialmente rural, tivesse maior acesso e consumo dos alimentos in natura, muitas vezes produzidos nas suas próprias propriedades.

Observou-se reduzido percentual dos adolescentes que consumiram frutas e verduras mais de três vezes na semana anterior a entrevista. Estudo realizado com 377 escolares da zona rural de Barão do Triunfo/RS ${ }^{22}$, o qual investigou a adesão aos 10 Passos para uma Alimentação Saudável ${ }^{24}$, mostrou uma adesão extremamente baixa ao passo três (consumo diário de frutas e verduras) por escolares, assemelhando-se aos achados desta investigação.

Outros estudos, realizados na cidade de São Paulo/SP, também encontraram resultados semelhantes ${ }^{25,26}$. Por conseguinte, o consumo de frutas e verduras é considerado fator de proteção contra doenças cardiovasculares, câncer, diabetes e constipação intestinal, devido à sua riqueza em vitaminas, minerais e fibras ${ }^{27}$. Presume-se que a compra de frutas e verduras, pela população estudada, seja difícil devido à dificuldade de acesso aos supermercados centrais e à monocultura utilizada pelos agricultores locais.

Quanto ao consumo dos marcadores de alimentação não saudável, constatou-se que a proporção de alunos que consumiam regularmente variou de $15,2 \%$ a $31,0 \%$, sendo menor a proporção daqueles que consumiam embutidos e maior daqueles que consumiam bolachas/biscoitos doces ou recheados e guloseimas. Levando em consideração os resultados anteriores sobre o baixo consumo de alimentos saudáveis, o resultado pode caracterizar um comportamento da zona rural, onde os moradores apresentam maiores frequências de alimentos básicos, como arroz e feijão.

O baixo consumo de embutidos pelos escolares investigados evidencia uma conduta alimentar saudável, a qual se associa à proteção para o desenvolvimento de hipertensão arterial e dislipidemia ${ }^{27}$. Um estudo realizado com escolares de São Luís/MA e outro que avaliou a saúde de escolares de todas as capitais brasileiras também demonstraram baixo consumo desse tipo de alimento por escolares, corroborando com os achados desta investigação $0^{9,28}$.

Constatou-se que a maioria dos escolares que trouxe lanche de casa consumiu alimentos considerados saudáveis, entretanto o consumo de alimentos considerados não saudáveis também foi elevado (Tabela 3). Esse dado sugere que ainda está presente a preocupação dos pais com o que o seu filho vai consumir na escola pelo fato deste levar a alimentação de casa, mas se sabe que, devido às demandas geradas pelo novo modo de vida, caracterizado pela escassez de tempo, vasta oferta de produtos, flexibilização dos horários das refeições e grande apelo publicitário em torno dos alimentos, estes acabam abrindo mão de um lanche de melhor qualidade preparado em casa. Essas mudanças no padrão alimentar da população rural ficam evidenciadas em estudos anteriores, como aquele realizado com famílias de alunos de uma escola da zona rural de Valinhos/ SP, que constatou que mesmo os agricultores já não consomem com a mesma frequência os alimentos que produzem (produtos considerados saudáveis), no qual 59,5\% relataram não consumir frutas, 70,9\% não consumiram verduras e $53,2 \%$ não consumiram nenhum legume no dia alimentar ${ }^{29}$.

Apesar dos pontos positivos apontados no início desta seção, o presente estudo apresenta algumas limitações. O instrumento utilizado para a coleta de dados foi um questionário autoaplicado e estruturado, nesse sentido e em razão das questões serem fechadas, pode ser que algum alimento consumido pelos escolares não estivesse contemplado no instrumento, além disso, como estes não estavam habituados com este tipo de investigação, podem ter ocorrido pequenos erros na estimativa do consumo (porções).

\section{Conclusão}

O presente estudo encontrou um elevado percentual de escolares de compravam lanche na cantina ou bar da escola e que consumiam a merenda fornecida pela instituição; além disso os escolares que levavam lanche de casa para a escola consumiram, na maioria dos dias da semana anterior, alimentos considerados saudáveis. Entretanto, o consumo de alimentos considerados saudá- 
veis entre os adolescentes escolares foi baixo, de modo que os alimentos saudáveis mais consumidos foram feijão e iogurte.

Nesse sentido, futuras intervenções de base escolar com foco na alimentação devem ser realizadas de acordo com as investigações realizadas na zona rural, com intuito de priorizar estratégias a fim de melhorar o quadro atual de práticas inadequadas de alimentação.

\section{Colaboradores}

CTR Bubolz contribuiu com análise dos dados, escrita do artigo e revisão final do mesmo; AJ Rombaldi contribuiu com a concepção do estudo, análise dos dados, escrita do artigo e revisão final; NG Gonzales contribuiu com a coleta dos dados, escrita do artigo, análise dos dados e revisão final; MR Azevedo contribuiu com a concepção do estudo, revisão e análise final do mesmo; SW Madruga contribuiu com a concepção do estudo, análise dos dados e revisão final. 


\section{Referências}

1. Fundo das Nações Unidas para a Infância (UNICEF). Adolescência: uma fase de oportunidades. Situação Mundial da Infância. Nova Iorque: UNICEF; 2011.

2. Brasil. Ministério da Saúde (MS). Saúde na escola. Brasília: MS; 2009.

3. Minas Gerais. Secretaria de Estado de Saúde (SES). Atenção à saúde do adolescente. Belo Horizonte: SAS/ MG; 2006.

4. Brasil. Ministério da Saúde (MS). Orientações básicas de atenção integral à saúde de adolescentes nas escolas e unidades básicas de saúde. Brasília: Editora do Ministério da Saúde; 2013.

5. Monteiro CA, Benicio MHD, Konno SC, Silva ACF, Lima ALL, Conde WL. Causas do declínio da desnutrição infantil no Brasil, 1996-2007. Rev Saude Publica 2009; 43(1):35-43.

6. Teixeira AS, Philippi ST, Leal GVS, Araki EL, Estima CCP, Guerreiro RER. Substituição de refeições por lanches em adolescentes. Rev Paul Pediatr 2012;30(3):330-337.

7. Carmo MB, Toral N, Silva MV, Slater B. Consumo de doces, refrigerantes e bebidas com adição de açúcar entre adolescentes da rede pública de ensino de Piracicaba, São Paulo. Rev Bras Epidemiol 2006; 9(1):121-130.

8. Toral N, Slater B, Silva MV. Consumo alimentar e excesso de peso de adolescentes de Piracicaba, São Paulo. Rev Nutr 2007; 20(5):449-459.

9. Conceição SIO, Santos CJN, Silva AAM, Silva JS, Oliveira TC. Consumo alimentar de escolares das redes pública e privada de ensino em São Luís, Maranhão. Rev Nutr 2010; 23(6):993-1004.

10. Toral N, Slater B, Cintra IP, Fisberg M. Comportamento alimentar de adolescentes em relação ao consumo de frutas e verduras. Rev Nutr 2006; 19(3):331-340.

11. Instituto Brasileiro de Geografia e Estatística (IBGE). Cidades. [acessado 2016 Jun 10]. Disponível em: http:// cidades.ibge.gov.br/xtras/perfil.php?lang $=\&$ codmun $=430450 \&$ search $=$ rio-grande-do-sul|cangucu

12. Callo G, Gigante DP, Barros FC, Horta BL. Excesso de peso/obesidade no ciclo da vida e composição corporal na idade adulta: coorte de nascimentos de Pelotas, Rio Grande do Sul, Brasil, 1982. Cad Saude Publica 2016; 32(4):e00174014.

13. Accioly E. A escola como promotora da alimentação saudável. Cien Tela 2009; (2)2:1-9.

14. Barbosa NVS, Machado NMV, Soares MCV, Pinto ARR. Alimentação na escola e autonomia - desafios e possibilidades. Cien Saude Colet 2013; 18(4):937-945.

15. Danelon MAS, Danelon MS, Silva MV. Serviços de alimentação destinados ao público escolar: análise da convivência do Programa de Alimentação Escolar e das cantinas. Segur Aliment Nutr 2006; 13(1):85-94.

16. Amorim NFA, Schmitz BAS, Rodrigues MLCF, Recine EGI, Gabriel CG. Implantação da cantina escolar saudável em escolas do Distrito Federal, Brasil. Rev Nutr 2012; 25(2):203-217.

17. Brasil. Ministério da Saúde (MS), Rede Internacional em Defesa do Direito de Amamentar - IBFAN. ENPACS: Estratégia Nacional Para Alimentação Complementar Saudável: Caderno Do Tutor. Brasília: MS; 2010.
18. Spohr CF, Fortes MO, Rombaldi AJ, Hallal PC, Azevedo MR. Atividade física e saúde na Educação Física escolar: efetividade de um ano do projeto "Educação Física +". Rev Bras Ativ Fis Saúde 2014; 19(3):300-313.

19. Brasil. Ministério da Saúde (MS). Protocolos do Sistema de Vigilância Alimentar e Nutricional - SISVAN na assistência à saúde. Brasília: MS; 2008.

20. Brasil. Ministério da Saúde (MS). Politica Nacional de Alimentação e Nutrição. Brasília: MS; 2012.

21. Brasil. Ministério da Saúde (MS). Regulamentação da comercialização de alimentos em escolas no Brasil: Experiências estaduais e municipais. Brasília: MS; 2007.

22. Raphaelli CO, Azevedo MR, Hallal PC. Associação Entre comportamentos de risco à saúde de pais e adolescentes em escolares de zona rural de um município do Sul do Brasil. Cad Saude Publica 2011; 27(12):24292440.

23. Neutzling MB, Assunção MCF, Malcon MC, Hallal PC, Menezes AMB. Hábitos Alimentares de Escolares adolescentes de Pelotas, Brasil. Rev Nutr 2010; 23(3):379388.

24. Brasil. Ministério da Saúde (MS). Dez passos para uma alimentação saudável: guia alimentar para crianças menores de dois anos: um guia para o profissional da saúde na atenção básica. $2^{\text {a }}$ ed. Brasília: MS; 2013.

25. Bigio RS, Junior EV, Castro MA, César CLG, Fisberg RM, Marchioni DML. Determinantes do consumo de frutas e hortaliças em adolescentes por regressão quantílica. Rev Saude Publica 2011; 45(3):448-456.

26. Garcia GCB, Gambardella AMD, Frutuoso MFP. Estado nutricional e consumo alimentar de adolescentes de um centro de juventude da cidade de São Paulo. Rev Nutr 2003; 16(1):41-50.

27. Brasil. Ministério da Saúde (MS). Guia alimentar para a população brasileira: promovendo a alimentação saudável. Brasília: MS; 2008.

28. Levy RB, Castro IRR, Cardoso LO, Tavares LF, Sardinha LMV, Gomes FS, Costa AWN. Consumo e comportamento alimentar entre adolescentes brasileiros: Pesquisa Nacional de Saúde do Escolar (PeNSE), 2009. Cien Saude Colet 2010; 15(Supl. 2):3085-3097.

29. Boog MCF, Fonseca MCP, Alves HJ, Voorpostel CR. Agricultores Consomem Frutas, Verduras e Legumes? Bases para Ações Educativas. Segur Aliment Nutr 2008; 15(2):85-97.

Artigo apresentado em 10/03/2016

Aprovado em 02/09/2016

Versão final apresentada em 04/09/2016 